\title{
A New Method of Basis Function's Pseudorandom Phase Generation in TDCS and Its Performance Analysis
}

\author{
Fangjian $\mathrm{Li}^{*}$
}

ChongQing College of Electronic Engineering, ChongQing, 401331, China

\begin{abstract}
Based on the requirements of the pseudorandom phase vector in transform domain communication system(TDCS), a new method using chaos mapping and m-sequences control to generate the pseudorandom phases of the basis function in TDCS was proposed. The implementation method and the simulation results regarding the correlation performance for the basis function were discussed in detail. Compared with that of the parallel, the basis function's randomicity of the new method is $\mathrm{N}$ times by that of one-ary method (the length of m-sequence is $\mathrm{N}$ ), as well the basis functions have better correlation performance for that many basis functions with small cross-correlation coefficients can be generated by chaos mapping. The simulation results show that the correlation performance of the basis function produced by the new method was improved and the performance on BER was better.
\end{abstract}

Keywords: TDCS, basis function, pseudorandom phase, m sequence, chaos mapping.

\section{INTRODUCTION}

Transform Domain Communication System (TDCS) as one of the candidate for cognitive radio, perception of environment spectrum in the frequency domain, from the point of view of waveform design, take the initiative to avoid interference, so that the system has good anti-jamming performance, at the same time by random phase mapping enables the system to have resistance to intercept and multiple access performance.

Differences between TDCS and traditional communication system is: first, through the design of TDCS waveform signals in the transform domain channel estimation: second, TDCS did not use the carrier modulation, but information modulated using basis functions in a similar noise.

TDCS is a new technology $[1,2]$ based on the development of cognitive radio, spread spectrum communication and transform domain technology. Through the spectrum sensing on the surroundings, the TDCS conducts interference evasion united with transceiver in transform domain. Depend on the performances of flexible frequency spectrum application, good anti-interference and low probability of intercept, the TDCS has gotten more and more attention [3]. Differences between TDCS and traditional communication system, first through TDCS transform domain channel estimation in the design of signal waveform, followed by TDCS without the use of carrier modulation, but information modulation.TDCS has strong anti-interference ability and high base function of a noise like LPI (LPI) characteristics.
The basic function design is a critical technology [4] to realize the anti-interference and low probability of intercept performance of TDCS. At present, the research on TDCS basic function design mainly focuses on the magnitude spectrum design and research on arithmetic of pseudo-random phase generation is relatively less [5-7]. In TDCS, it is the pseudo-random phase application in the basic function, the low probability of the system can finally be realized, and thus the basic function is required to have good randomness and correlation. In order to solve this problem, the document [8] proposes the adoption of double $\mathrm{M}$ sequence to control the phase mapping methods of basic function, which strengthen the randomness of basic function; the document [9] implements chaos mapping to realize a better correlation of basic function. This paper bases on the specific introduction of TDCS principles and basic function design thoughts, combined with the advantages of two methods in document [8] and document [9], to propose a method of utilizing M sequence control method in chaos mapping to generate random phase of basic function. Meanwhile, the performance of basic function is analyzed in detailed and finally, the simulation of error rate of the system is conducted, which verifies the effectiveness of the improved method (Fig. 1).

\section{TDCS PRINCIPLE}

The TDCS system transmitter conducts sampling on the surroundings and threshold decision by spectrum sensing and generates a free spectrum mark vector, recorded as $\mathrm{A}=\{\mathrm{A} 0, \mathrm{~A} 1, \mathrm{~A} 2, \ldots, \mathrm{AN}-1\}$, the element $\mathrm{Ak}$ is 1 or 0 , which indicates the availability or unavailability of frequency range $\mathrm{k}$ respectively, thus the occupied frequency range can be avoided to reach the purpose of anti-interference. Meanwhile, through the phase spectrum generated by 


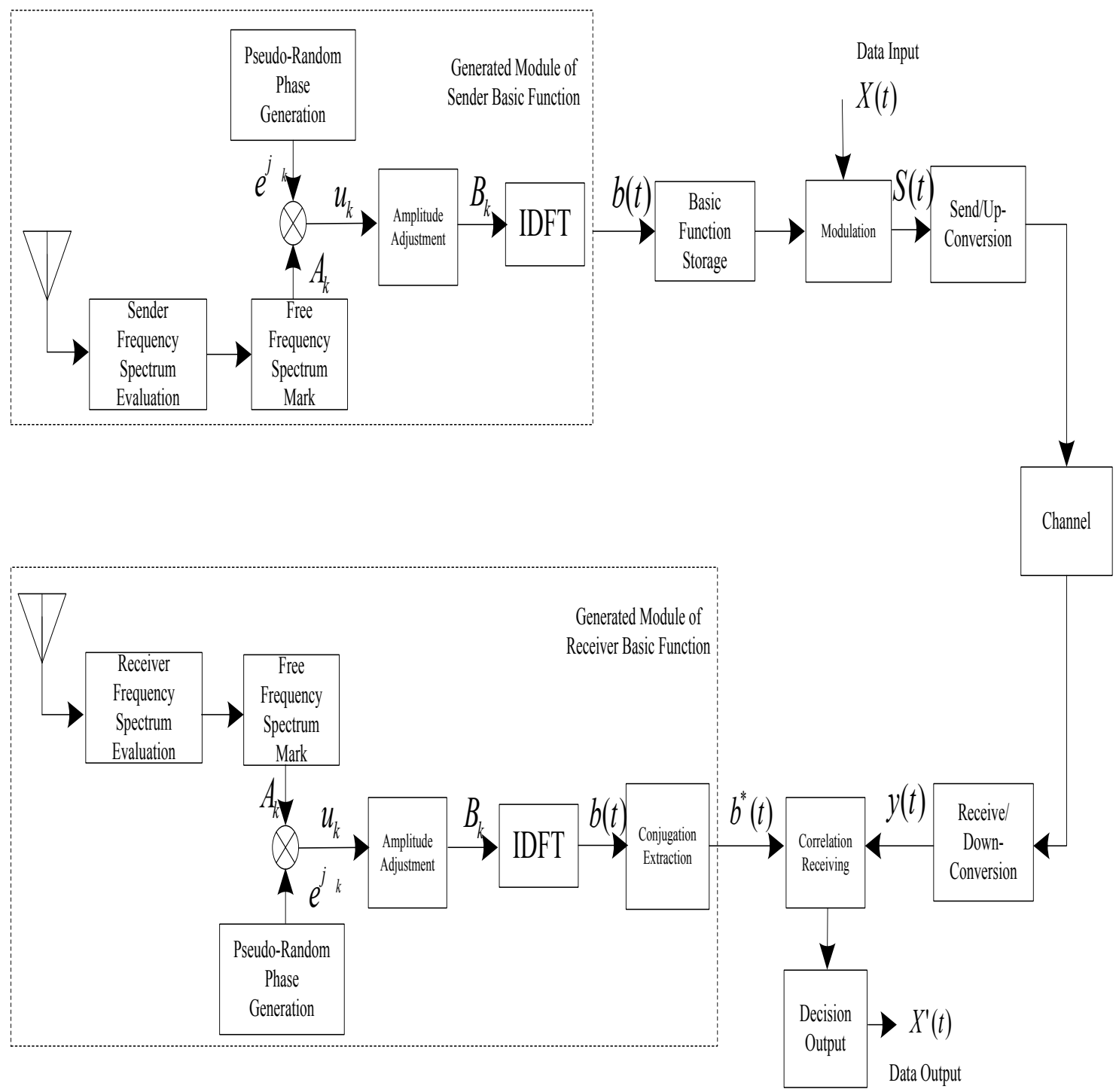

Fig. (1). TDCS functional block diagram.

pseudo-random phase generator, this vector phase is in accordance with the uniform random distribution in $[0,2 \pi]$ and has the same length with the phase mark length [10]. The frequency domain vector of orthogonal basic function between frequency spectrum and interference spectrum is gotten through the corresponding multiplication of phase spectrum generated by generator and free frequency spectrum mark vector element and then certain amplitude adjustment is conducted according to the requirements of transmitted power. Then, the vector is applied with IDFT and the time domain formation of basic function used in modulation is: . The modulation is conducted by basic function after data input and then the data outputs with power amplifier and antenna. The receiver adopts the same basic function generation method. Suppose the electromagnetic environment of receiving and transmitting is the same, the generated basic function will be the same. After synchronization, the receiving end can adopt time domain correlation reception and restore the data sending.

\section{RANDOM PHASE GENERATION BY SEQUENCE M CONTROL METHOD OF CHAOS MAPPING}

The performance of basic function is correlated with the PN sequence used in random phase mapping [11]. The selection and design of PN sequence is the same with spreading code, which not only requires a relative good self-correlation property (namely a relative large autocorrelation peak), but also has a relative good cross-correlation property (namely the cross-correlation peak is very small and verges to 0 ). Meanwhile, the TDCS multiple access capability is ensured through random phase mapping and different $\mathrm{PN}$ sequence can work as different address code of users. Traditional TDCS system generates random phase through sequence $m$. Two main problems exist while generating random phase by sequence $\mathrm{m}$ : firstly, the quantity of sequence $\mathrm{m}$ is relative small, which cannot satisfy the customer demand of multiple access communication; secondly, the cross-correlation property is relative poor (which requires a careful selection for 


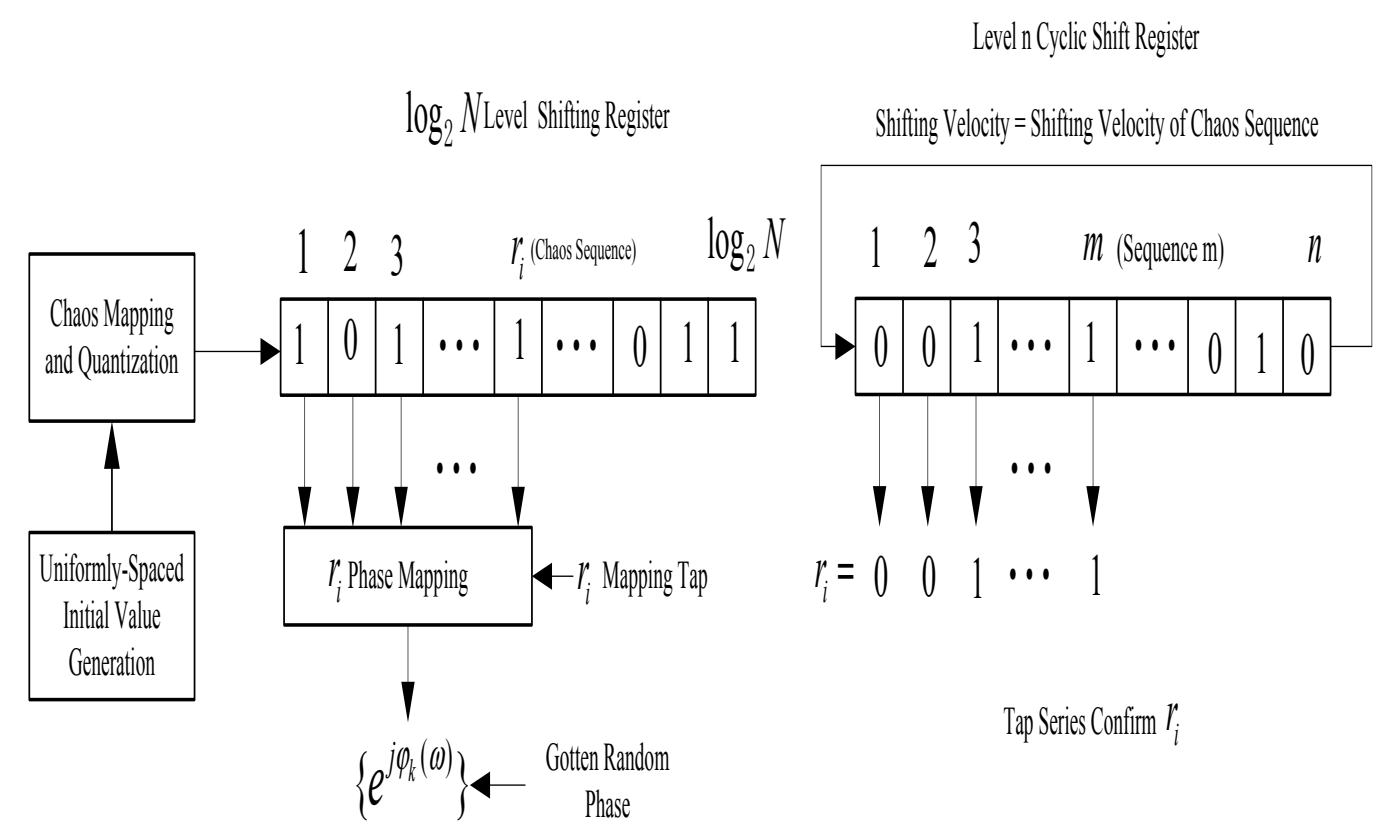

Fig. (2). Random phase generator based on sequence m control method of chaos mapping.

different sequence $\mathrm{m}$ that have better cross-correlation performance), which will generate interference while multiple access application.

In order to solve the two problems above, this paper constructs the random phase mapping by utilizing chaos sequence. The chaos sequence has favorable noise characteristic and ideal self-correlation and cross-correlation property. At the same time, the random sequences can be generated in large quantity by the iteration with different initial value, which is convenient for multiple access application. The chaos sequence adopts improved Logistic mapping [12] and the definition is given by formula (1):

$x_{k+1}=1-2\left(x_{k}\right)^{2},-1<x_{k}<1$

The self-correlation function of sequence is:

$$
R(m)=\lim _{N \rightarrow \infty} \frac{1}{N} \sum_{k=0}^{N-1} x_{k} x_{k+m}-\bar{x}=\left\{\begin{array}{cc}
0.5, & m=0 \\
0, & m \neq 0
\end{array}\right.
$$

The $\bar{x}$ in formula is a mean value. The cross-correlation function of the sequence is:

$$
R_{1,2}(m)=\lim _{N \rightarrow \infty} \frac{1}{N} \sum_{k=0}^{N-1}\left(x_{1 k}-\bar{x}\right)\left(x_{2(k+m)}-\bar{x}\right)=0
$$

The value of full-mapping chaos sequence is uniformly distributed in $[-1,1]$. For digitalization application, two-value quantification processing is conducted, namely:

$y_{k}=\left\{\begin{array}{l}0, x_{k}<0 \\ 1, x_{k} \geq 0\end{array}\right.$

The two-value sequence $y_{k}$ is generated through the processing above and the phase mapping is conducted with $y_{k}$.
For chaos sequence, different initial value will generate different sequence. Therefore, the chaos sequence generated by different initial value can be distributed to different users as the address code of multiple accesses. For this, this paper adopts equi-spaced method in initial value sequence generation. Meanwhile, because the infiniteness of chaos sequence, $2^{r_{i}}$ complex value random phase should be generated and the generated chaos sequence can be shifted into a level $\mathrm{n}$ shifting register successively. $r_{i}$ taps are selected in level $\mathrm{n}$ shifting register to construct random phase mapper and the phase mapping length in chaos sequence is the sampling number $N$ in basic function. The chaos sequence method above is expected to improve the correlation between the basic function frequency spectrum and the following will discuss the improvement on its randomness.

The randomness of the basic function mainly correlates with the $r$ input in the phase mapper and the $r$ is fixed. Provided the $r$ is given, the total number of the random phase will keep fixed in one cycle in a unit circle. In the meantime, the randomness of the phase directly decides the randomness of the basic function. In order to improve the phase randomness of basic function, this paper utilizes $r$ tap decided by the $M$ sequence to control the chaos sequence and by changing the $r$ that inputs into the phase mapper, the phase randomness of the basic function can thus be improved.

In conclusion, the improvement as Diagram 2 is conducted on the traditional TDCS phase mapping scheme. In (Fig. 2), $m$ is the series of shifting register that generates sequence $\mathrm{m}$; the series of the phase $r_{i}$ is decided by the value of level $m$ shifting register of sequence $m$. If the value of the previous $\mathrm{m}$ shifting register is $r_{i}=4$, then the chaos sequence has level 4 mapping tap and the value of previous $r_{i}$ shifting register (namely, 4) of chaos sequence correspond- 


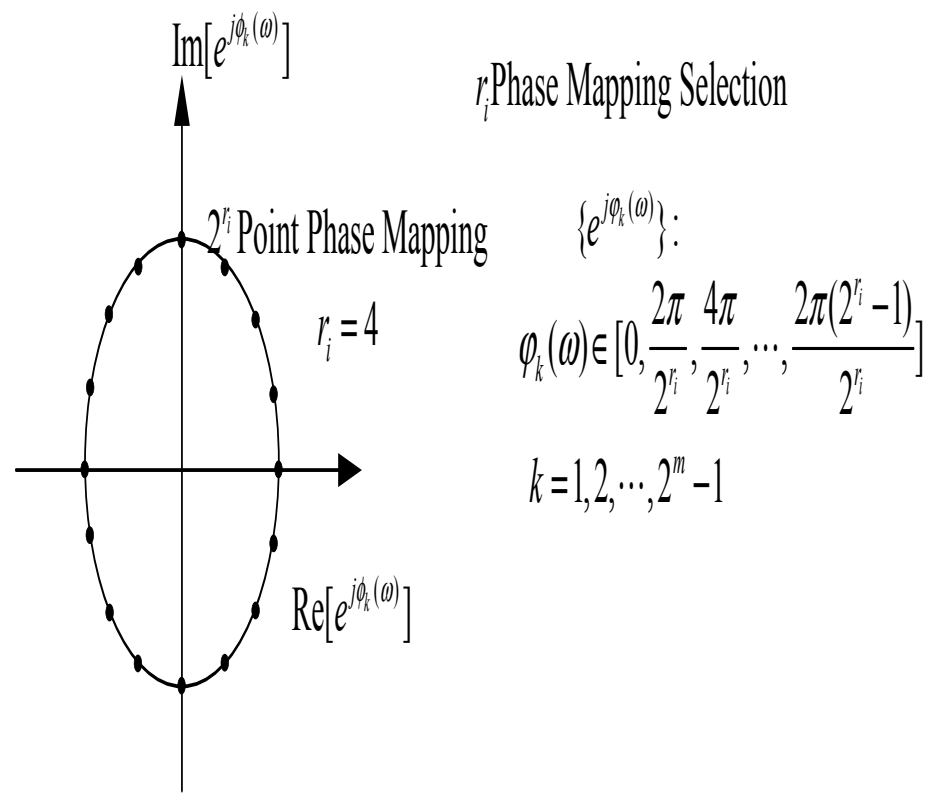

Fig. (3). $r_{i}$ Phase mapping methods.

ing to the tap decides the phase mapping with a selectable phase points of $2^{r_{i}}=16$ as shown in (Fig. 3).

\section{PERFORMANCE ANALYSIS OF BASIC FUNC- TION}

\subsection{Basic Function Randomness Analysis}

The randomness of basic function in time domain is decided by $A_{k}$ and $\varphi_{k}$, and $\varphi_{k}$ is decided by both chaos sequence and sequence $\mathrm{m}$. The basic functions generated by different chaos sequence and sequence $m$ have different randomness, namely has different level of similarity with noise. $A_{k}$ was decided by the distribution of free frequency spectrum and when the free frequency spectrum is uniformly distributed, the basic function has better randomness [13].

In sequence $\mathrm{m}$ control method of chaos mapping, the chaos sequence has different shifting velocity compared with sequence $\mathrm{m}$. Each time the chaos sequence conducts shifting for $N$ times, sequence $m$ will shift for once, namely the generation of each TDCS data symbol is decided by the current value of sequence register $m$ and the $N$ th time phase mapping in chaos sequence. Meanwhile, the value number of sequence register $\mathrm{m}$ is the length $N_{m}\left(N_{m}=2^{m}-1\right)$, thus the selectable data symbol number of TDCS is the value number of the shifting register of sequence $\mathrm{m}$. Therefore, the following can be concluded:

$r_{i} \in\{1,2, \cdots, N\} \quad i=1,2, \cdots, N$

Because the correlation has been improved by applying chaos sequence mapping and the phase of the basic function is decided by both the $m$ and $r_{i}$, the randomness in basic function generation has been improved and singledimensional controls perimeter is added compared with the traditional sequence $\mathrm{m}$ method. For the given $\mathrm{r}$, $C_{n}^{r}=n ! /(r !(n-r) !)$ types of basic functions can be generated by mapping. If $r$ is input into random phase mapper, the number of generated basic functions is:

$N_{r}=\sum_{r=1}^{n} C_{n}^{r}=C_{n}^{1}+C_{n}^{2}+\ldots+C_{n}^{n}=2^{n}-1$

If the selectable quantity of chaos sequence is $N_{c}$ and the series of shifting register of sequence $\mathrm{m}$ is $m$, then the total phase mapping is:

$N_{s}=\left(2^{m}-1\right) N_{c}=N_{m} N_{c}$

The detection probability will be $1 /\left(2^{m}-1\right)$ of the detection probability that generated by pseudo-random phase generation method of traditional single-dimensional sequence $\mathrm{m}$. Along with the increasing of $m$, the detection probability presents an exponential fall.

\subsection{Correlation Analysis of Basic Function}

The correlation function of the two time domain basic functions $b_{l}(n)$ and $b_{q}^{*}(n)$ with same length is:

$$
\begin{aligned}
& R(m)=E\left[b_{l}(n) b_{q}^{*}(n+m)\right] \\
& =\sum_{n=0}^{N-1-m}\left(\frac{1}{N} \sum_{u=0}^{N-1} A_{u} e^{\phi_{u}} e^{j \frac{2 \pi m u}{N}}\right) \cdot\left(\frac{1}{N} \sum_{v=0}^{N-1} A_{v}^{*} e^{-\phi_{v}} e^{-j \frac{2 \pi(n+m) v}{N}}\right) \\
& =\frac{1}{N^{2}} \sum_{n=0}^{N-1-m} \sum_{u=0}^{N-1} \sum_{v=0}^{N-1} A_{u} A_{v}^{*} e^{\phi_{u}-\phi_{v}} e^{j \frac{j \pi(n u-n v-m v)}{N}}
\end{aligned}
$$




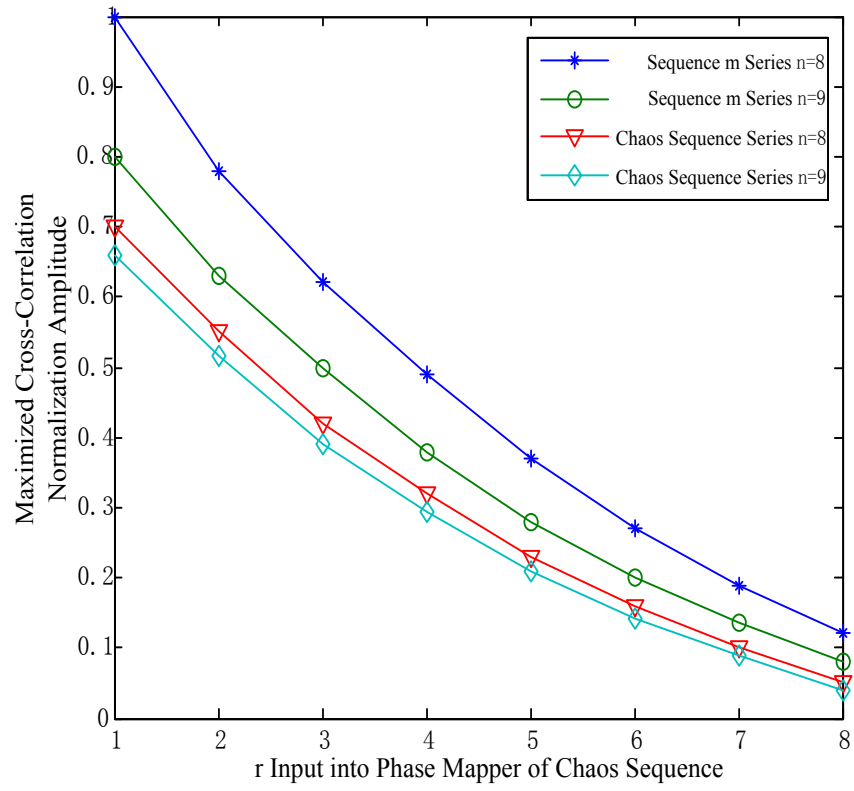

Fig. (4). Maximized cross-correlation value of basic function gotten by the adoption of sequence $\mathrm{m}$ and $\mathrm{r}$ input into random phase mapper of chaos sequence.

Among them, $N$ is the sampling number of basic function, $m$ is the time shifting between the basic functions, $A_{u}$ and $A_{v}^{*}$ are the frequency spectrum amplitude of the two basic functions, $\phi_{u}$ and $\phi_{v}$ are the phase of the $u^{\text {th }}$ and $v^{\text {th }}$ subcarriers of the two basic functions respectively and $S_{l}$ and $S_{q}$ represent the modulating data of the two basic functions respectively.

When $l \neq q$ and $m=0$, the maximized value of the basic function cross-correlation function is:

$R_{\text {cross }}(0)=\frac{1}{N^{2}} \sum_{u=0}^{N-1}\left|A_{u}\right|^{2} e^{\phi_{u}-\phi_{v}}$

When $l=q$ and $m \neq 0$, the self-correlation function of the basic function is:

$R_{\text {auto }}(m)=\frac{1}{N^{2}} \sum_{u=0}^{N-1}\left|A_{u}\right|^{2} e^{j \frac{2 \pi m u}{N}}$

When $m=0$, the maximized value of the basic function self-correlation function is:

$$
R_{\text {auto }}(0)=\frac{1}{N} \sum_{u=0}^{N-1}\left|A_{u}\right|^{2}
$$

\section{SIMULATION RESULT}

In Fig. (4), the maximized cross-correlation value of basic function gotten by the sequence $\mathrm{m}$ adoption and $\mathrm{r}$ input into chaos sequence random phase mapper is shown under the improved scheme of random phase. From the diagram, it is known that when $r$ is already known, the maximized value of cross-correlation function of basic function will decrease as the series increasing of sequence $m$ or chaos sequence, which is because the larger the sequence series becomes, the more random phase number can be gotten and then the generated basic function can have better randomness finally and its cross-correlation will become weaker. However, under the same series, the maximized cross-correlation value of chaos sequence will be smaller than sequence $\mathrm{m}$; when the sequence series is already known, the cross-correlation maximized value of basic function will decrease along with the increasing of $r$, because the increasing of $r$ makes the number of random phase in one cycle in a unit circle increase and also intensifies the basic function randomness and weakens its cross-correlation.

As are shown in theoretical analysis and simulation (Fig. 4), the proposed random phase mapping improvement scheme can effectively improve the correlation property of basic functions. Because the generated basic function is similar with the signal of white noise and has selforthogonality, namely the cross-correlation value of different time-shift waveforms in basic functions is very small. Therefore, in the modulation unit of TDCS, the modulation can be conducted by CSK (Cyclic Shift Keying), namely to utilize the favorable correlation property of basic function to represent different code elements with different time-shift waveforms in basic functions. In addition, bipolar modulation can be adopted, namely the different binary codes can be represented by basic function and the negative number of basic function. The simulation analysis compares the modulation performance of traditional TDCS system with the one in improved TDCS system to verify the effectiveness of the improved scheme.

There are two modulation methods in TDCS: bipolar modulation and CSK modulation. In AWGN channel, the modulation transmission error rates of bipolar modulation and MCSK modulation in TDCS system are: 


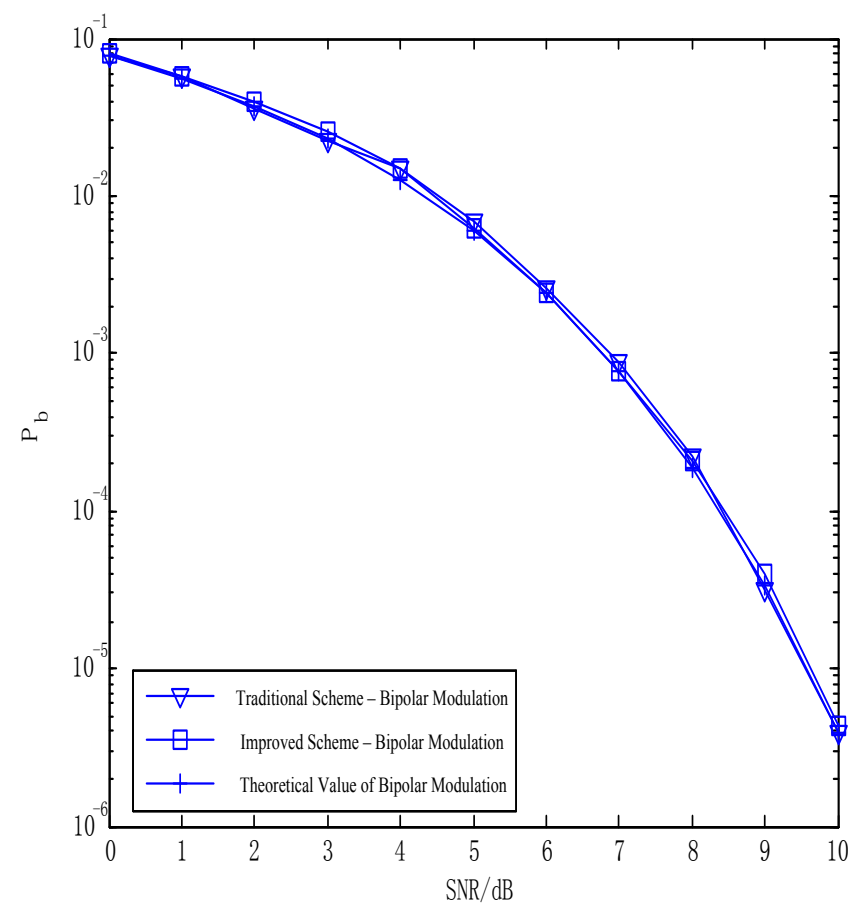

Fig. (5). TDCS system performance comparison in the adoption of bipolar modulation improvement.

$$
P_{b}=Q\left(\sqrt{\frac{\zeta E_{b}}{N_{0}}}\right)
$$

$P_{b}(M) \leq(M-1) Q\left(\sqrt{\frac{E_{b} \log _{2}(M)}{N_{0}}}\right)$

Among them, the $\zeta=2$ corresponds to bipolar modulation and $\zeta=1$ corresponds to the BCSK.

For the bipolar modulation TDCS system, in the simulation scheme, if all the frequency spectrum is available, namely the free frequency spectrum mark vector element is all equal to 1; channel model: additive white Gaussian noise; SNR (signal to noise ratio): $0 \sim 10 \mathrm{~dB}$; the traditional scheme adopts the sequence $m$ of level $n=9$; random phase mapper: $\mathrm{r}=3$ and for the random phase mapper in improved scheme adopts the chaos sequence of level $\mathrm{n}=9$; random phase mapper: $r \in[1,9]$. The simulation results are shown in (Fig. 5). From (Fig. 5), the simulation value of bipolar modulation is basically the same with theory and the bipolar modulation performance keeps the same after the improvements, because it modulates the data information by the positive and negative waveforms in basic function and has no special requirements on the correlation of basic function.

For the TDCS system with CSK modulation, the simulation scheme is the same with Fig 5 and the results are shown in (Fig. 6). From (Fig. 6), BCSK modulation simulation value is a little bit higher than theoretical value, because the relation between the CSK symbols are not absolute orthogonal. Meanwhile, the simulation values of $8 \mathrm{CSK}$ and $16 \mathrm{CSK}$ modulation error rates is a little bit lower than the theoretical value, because the theoretical values of error rates in multiple CSK modulation are the upper bound values of the real error rates. From the diagram, although the error rate of the BCSK is higher than bipolar modulation, the CSK have the ability of multiple modulations, which can realize multiple modulations and improve the error rates performance to improve the transmission rate. At the same time, the improved BCSK modulation performance is closer to the theoretical value compared with the previous one, because the BCSK utilizes the correlation property of basic function to conduct modulation. Besides, the relation between CSK signals are not complete orthogonal, namely the value of crosscorrelation is equal to 0 . Compared with the unimproved one, the improved one has better randomness of CSK signal phase, which improves its correlation property, namely the cross-correlation value approaches to 0 , thus the system performance is improved.

\section{CONCLUSION}

This paper proposes a basic function phase generation method of sequence $m$ control method of chaos mapping. By the analysis and simulation on the basic function generated by the traditional phase mapping method, it is proved that the basic function and its self-correlation function generated by sequence $m$ control method of chaos mapping have ideal properties, which can fit the function requirements of the noise-like generated in TDCS. By this property, the CSK data modulation can be realized, the method of which adds single-dimensional sequence $\mathrm{m}$ control perimeter compared with the phase mapping generation method of chaos sequence control and the basic function generated has better randomness. The degree of randomness increasing presents 


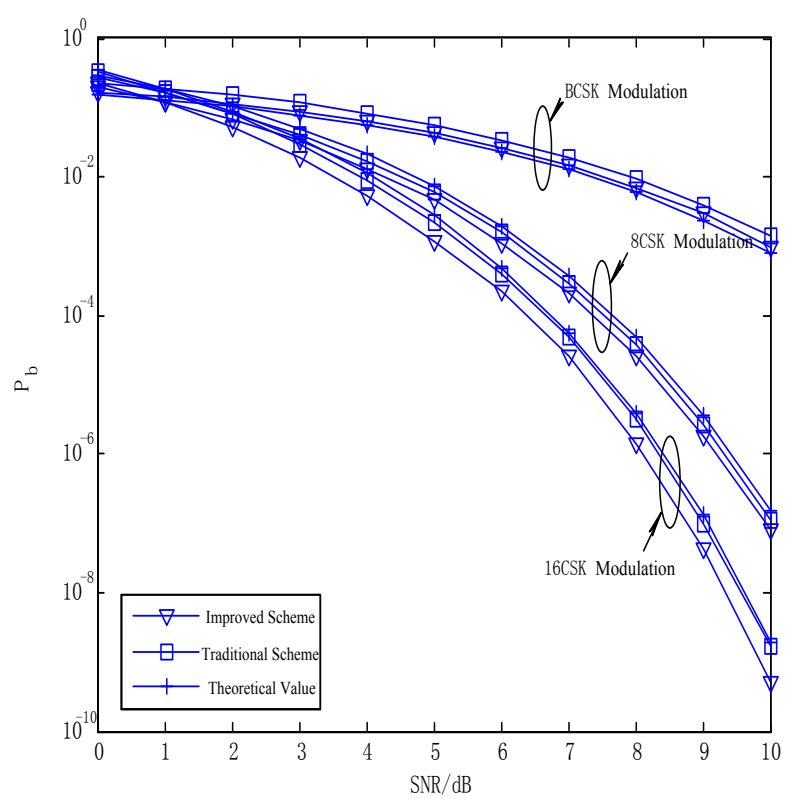

Fig. (6). TDCS system performance comparison in the adoption of CSK mdulation improvement.

an exponential increase along with the length increase of sequence $\mathrm{m}$ applied. Because the chaos sequence phase mapping is adopted, which has better basic function crosscorrelation property compared with the one generated by traditional phase mapping of sequence $\mathrm{m}$, simulation results show that the basic function randomness and correlation property generated by the phase mapping method controlled by chaos mapping sequence $m$ is more favorable, which improves the modulation performance of the system and possesses the features of simplicity and being easy to realize.

\section{CONFLICT OF INTEREST}

The author confirms that this article content has no conflict of interest.

\section{ACKNOWLEDGEMENTS}

Declared none.

\section{REFERENCES}

[1] S.B. Wang, Z. Zhou, H.F. Dou, and K. Kwak, "A novel pulse for CR-UWB using compressed chirp waveforms and modified TDCS", In IEEE International Conference on Future Computer and Communication, 2010, pp. 541-545.

[2] H.X. Sun, G.A. Bi, Y.L. Guan, and Y. Shi, "Novel pseudorandom phase generation in transform domain communication systems", In Proceedings of International Conference on Innovations in Information Technology, 2011, pp. 18-22.

[3] R.L. Zhou, Q. Han, Reginald Cooper, V. Chakravarthy, and W. Zhiqiang, "A software defined radio based adaptive interference avoidance TDCS cognitive radio", In: IEEE International Conference On Communication, 2010, pp. 1-5.

[4] Zeng Wu, Da Xinyu and Shen Yong, "TDCS basic function generation and performance analysis based on multistage threshold decisions", College Journal of Chongqing University of Post and Telecommunication (Natural Science Section), vol. 24, no. 3, pp. 354357, 2012.

[5] C. Zhang, L. Xue and E. Zhang, "Transform domain narrow-band interference suppression based on self-adaption multi-threshold arithmetic", Academic Journal of Electron and Information, vol. 28, no. 3, pp. 461-464, 2006.

[6] R. Guosheng, M. Jun and Y. Xiao, "Fractal threshold research on transform domain communication system", Academic Journal of System Simulation, vol. 21, no. 13, pp. 4077-4083, 2009.

[7] C.D. Wang, Z.P. Zhang and S.Q. Li, "Interference avoidance using fractional fourier transform in transform domain communication system", In: The $9^{\text {th }}$ International Conference Advanced Communication Technology, Phoneix :ICACT 2007, no. 3, pp. 1756-1760.

[8] C. Wang, Z. Zhang and $\mathrm{S}$. Li, "A new basic function generation method and performance analysis", Academic Journal of University of Electronic Science and Technology of China, vol. 35, no. 4, pp. 648-652, 2006.

[9] S. He, Y. Ji and H. Pan, "Chaos generation method of random phase in TDCS", College Journal of Chongqing University of Post and Telecommunication, vol. 35, no. 12, pp. 1381-1385, 2008.

[10] M.L. Roberts, M.A, Temple, R.F. Mills and R.A, "Raines. Interference suppression characterization for spectrally modulated, spectrally encoded signals", IEE Electronic Letters, vol. 42, no. 19, pp. 1103-1104, 2006.

[11] S.A. Nunez, "Interference Suppression in Multiple Access Communications Using M-ary Phase Shift Keying Generated Using Spectral Encoding", Wright-Patterson AFB OH: Air Force Institute of Technology (AU), 2004.

[12] L. Hui, "Chaotic Digital Communication. Beijing": Tsinghua University press, 2006.

[13] C. Han, J. Wang and Y. Yang, "Addressing the control channel design problem: OFDM-based transform domain communication system in cognitive radio", Computer Networks, vol. 52, pp. 795$815,2008$. 